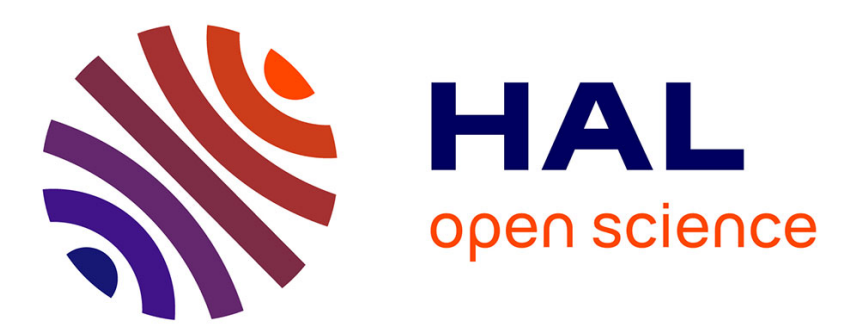

\title{
On the Complexity of Sets of Free Lines and Line Segments Among Balls in Three Dimensions
}

\author{
Marc Glisse, Sylvain Lazard
}

\section{To cite this version:}

Marc Glisse, Sylvain Lazard. On the Complexity of Sets of Free Lines and Line Segments Among Balls in Three Dimensions. Discrete and Computational Geometry, 2012, 47 (4), pp.756-772. 10.1007/s00454-012-9414-8. hal-00643880

\section{HAL Id: hal-00643880 \\ https://hal.inria.fr/hal-00643880}

Submitted on 23 Nov 2011

HAL is a multi-disciplinary open access archive for the deposit and dissemination of scientific research documents, whether they are published or not. The documents may come from teaching and research institutions in France or abroad, or from public or private research centers.
L'archive ouverte pluridisciplinaire HAL, est destinée au dépôt et à la diffusion de documents scientifiques de niveau recherche, publiés ou non, émanant des établissements d'enseignement et de recherche français ou étrangers, des laboratoires publics ou privés. 


\title{
On the Complexity of Sets of Free Lines and Line Segments Among Balls in Three Dimensions
}

\author{
Marc Glisse* $\quad$ Sylvain Lazard ${ }^{\dagger}$
}

September 30, 2011

\begin{abstract}
We present two new fundamental lower bounds on the worst-case combinatorial complexity of sets of free lines and sets of maximal free line segments in the presence of balls in three dimensions.

We first prove that the set of maximal non-occluded line segments among $n$ disjoint unit balls has complexity $\Omega\left(n^{4}\right)$, which matches the trivial $O\left(n^{4}\right)$ upper bound. This improves the trivial $\Omega\left(n^{2}\right)$ bound and also the $\Omega\left(n^{3}\right)$ lower bound for the restricted setting of arbitrary-size balls [Devillers and Ramos, 2001]. This result settles, negatively, the natural conjecture that this set of line segments, or, equivalently, the visibility complex, has smaller worst-case complexity for disjoint fat objects than for skinny triangles.

We also prove an $\Omega\left(n^{3}\right)$ lower bound on the complexity of the set of non-occluded lines among $n$ balls of arbitrary radii, improving on the trivial $\Omega\left(n^{2}\right)$ bound. This new bound almost matches the recent $O\left(n^{3+\varepsilon}\right)$ upper bound [Rubin, 2010].
\end{abstract}

\section{Introduction}

Given a set of objects in $\mathbb{R}^{3}$, a line is said to be free if it does not intersect the interior of any object (we assume here that all objects have a non-empty interior). A maximal free line segment is a (possibly infinite) segment that does not intersect the interior of any object and is not contained in any other segment satisfying the same property. We are interested here in the worst-case combinatorial complexity of sets of free lines, and sets of maximal free line segments.

Free lines and line segments play an important role in several topics in computational and combinatorial geometry. In particular, they play a central role in $3 \mathrm{D}$ visibility problems, such as the problem of determining the occlusion between two objects in a three-dimensional scene. In many applications, visibility computations are well-known to account for a significant portion of the total computation cost. Consequently, a large body of research is devoted to speeding up visibility computations through the use of data structures (see [14] for a survey). One such structure, the visibility complex [15, 23, encodes visibility relations by, roughly speaking, partitioning the set of maximal free line segments into connected components of segments tangent to the same set of objects. The vertices of this structure correspond, generically, to the maximal free line segments that are tangent to four objects in the scene, and the total number of faces, from dimension zero

${ }^{*}$ INRIA Saclay Île de France, Orsay, France. marc.glisse@inria.fr

${ }^{\dagger}$ INRIA Nancy Grand Est, LORIA laboratory, Nancy, France. sylvain.lazard@inria.fr 


\begin{tabular}{|c|cc|c|}
\hline & \multicolumn{2}{|c|}{ Free lines } & Free line segments \\
\hline Triangles & \multicolumn{2}{|c|}{$\Theta\left(n^{4}\right)$} & $\Theta\left(n^{4}\right)$ \\
\hline $\begin{array}{c}\text { Polyhedral } \\
\text { terrain }\end{array}$ & $\Omega\left(n^{3} 2^{c \sqrt{\log n}}\right)$ & $O\left(n^{4}\right)$ & $\Theta\left(n^{4}\right)$ \\
\hline $\begin{array}{c}n \text { disjoint homoth. } \\
\text { polytopes }\end{array}$ & $\Omega\left(n^{3}\right)$ & $O\left(n^{2} \lambda_{4}(n)\right)$ & $\Theta\left(n^{4}\right)$ \\
\hline $\begin{array}{c}k \text { polytopes } \\
\text { of total size } n\end{array}$ & $\Omega\left(n^{2}+n k^{3}\right)$ & $O\left(n^{2} k^{2}\right)$ & $\Theta\left(n^{2} k^{2}\right)$ \\
\hline \hline Unit balls & $\Omega\left(n^{2}\right)$ & $O\left(n^{3+\varepsilon}\right)$ & $\Theta\left(\mathbf{n}^{4}\right)$ \\
\hline Arbitrary balls & $\Omega\left(\mathbf{n}^{3}\right)$ & $O\left(n^{3+\varepsilon}\right)$ & $\boldsymbol{\Theta}\left(\mathbf{n}^{4}\right)$ \\
\hline
\end{tabular}

Table 1: Known bounds on the worst-case combinatorial complexity of sets of free lines and maximal free line segments (results presented in this paper are shown in bold).

to four, is exactly the combinatorial complexity of the space of maximal free line segments. The space of free lines in the presence of balls is also closely related, as noted by Agarwal et al. [1], to motion planning of a line among balls, or, equivalently, of a cylindrical robot (of infinite length) moving among points or balls. This is also related to computing largest empty cylinders among points in three dimensions, ray shooting, and other problems in geometric optimization.

Previous work. For scenes where the objects are $n$ triangles, the worst-case complexity of the space of free lines (or lines, for short) or maximal free line segments (or segments, for short) can easily be seen to be $\Theta\left(n^{4}\right)\left[8\right.$. When the triangles form a terrain, the same bound of $\Theta\left(n^{4}\right)$ holds for segments [9] and a near-cubic lower bound was proved for lines by Halperin and Sharir [17] and Pellegrini [22]. De Berg et al. [10] showed an $\Omega\left(n^{3}\right)$ lower bound and an almost matching $O\left(n^{2} \lambda_{4}(n)\right)$ upper bound ${ }^{1}$ on the complexity of the set of free lines among $n$ disjoint homothetic polytopes (i.e., convex polyhedra) of constant complexity. For such objects, a construction similar to the one for arbitrary triangles (see Figure 1) yields that the complexity of the set of free segments is also $\Theta\left(n^{4}\right)$.

When the triangles are organized into $k$ polytopes of total complexity $n$, with $k \ll n$, better bounds can be obtained. For the case of disjoint polytopes in general position, Efrat et al. [16] proved a worst-case bound of $O\left(n^{2} k^{2}\right)$ on the complexity of the set of free segments. When the $k$ polytopes may intersect, Brönnimann et al. [5] proved, independently, the tight bound of $\Theta\left(n^{2} k^{2}\right)$; their lower bound holds for disjoint polytopes, and their upper bound extends to polytopes in degenerate configurations. Any upper bound on the complexity of the set of segments trivially holds for lines as well. Thus, for free lines among $k$ polytopes of total complexity $n$, the upper bound of $O\left(n^{2} k^{2}\right)$ holds. However, the best known lower bound is $\Omega\left(n^{2}+n k^{3}\right)$, in which $\Omega\left(n^{2}\right)$ follows from the bound of $\Omega\left(n^{2} k^{2}\right)$ on maximal free line segments for $k=4$, and $\Omega\left(n k^{3}\right)$ can be obtained by slightly modifying the lower-bound construction [12, Th. 9] proving that the umbra cast on a plane by one segment light source in the presence of $k$ disjoint polytopes of total complexity $n$ can have $\Omega\left(n k^{2}\right)$ connected components (one simply has to consider $k$ perturbed copies of the segment light source).

\footnotetext{
${ }^{1}$ Recall that $\lambda_{4}(n)$ denotes an almost linear function equal to the maximum length of an $(n, 4)$-Davenport-Schinzel sequence [3].
} 


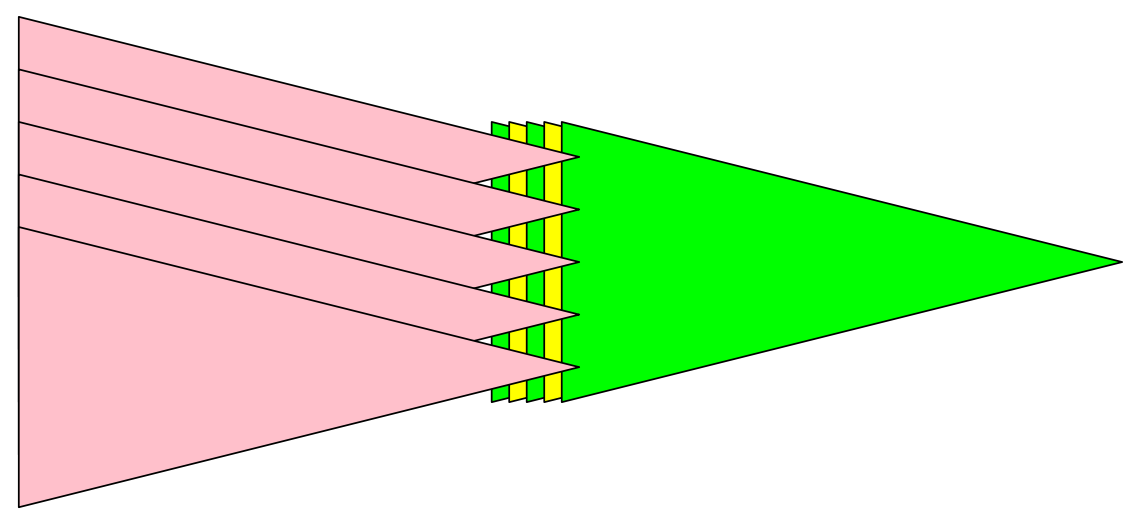

Figure 1: Two copies of such a set of $n$ identical triangles induce an $\Omega\left(n^{4}\right)$ bound on the complexity of the set of maximal free line segments.

Much less is known for curved objects. For $n$ unit balls, Agarwal et al. [1 proved an upper bound of $O\left(n^{3+\varepsilon}\right)$, for any $\varepsilon>0$, on the complexity of the space of free lines. Rubin [24] recently extended this result to balls of arbitrary radii. Devillers et al. [13] showed a simple bound of $\Omega\left(n^{2}\right)$ on the number of vertices of this free space (note that a trivial $\Omega\left(n^{2}\right)$ bound on the complexity of the whole space is obtained by considering sparsely distributed balls on two parallel planes). For $n$ balls of arbitrary radii, Devillers and Ramos (personal communication 2001, see also [13]) showed an $\Omega\left(n^{3}\right)$ lower bound on the complexity of the set of free line segments and the trivial upper bound of $O\left(n^{4}\right)$ holds.

The complexity of the space of maximal free line segments has also been studied in a random setting. Devillers et al. [13] proved that, in the presence of uniformly distributed unit balls, this structure has complexity $\Theta(n)$.

Related literature on free lines and line segments among objects fall in various categories. One deals with characterizing sets of lines tangent to four objects, such as balls or triangles, possibly in degenerate configuration (see [4, 6, 7, 19, 20, 21]). Another related line of research focuses on sets of lines that intersect objects and, in particular, on the complexity of the space of line transversals to a set of objects. For $n$ balls, Agarwal, et al. [2] showed an $\Omega\left(n^{3}\right)$ lower bound and a $O\left(n^{3+\varepsilon}\right)$ upper bound. For $k$ polytopes of total complexity $n$, Kaplan et al. [18] recently proved a $O\left(n^{2} k^{1+\varepsilon}\right)$ upper bound.

Our results. Our main contribution is a tight worst-case bound of $\Theta\left(n^{4}\right)$ on the space of maximal free line segments among unit balls, or, equivalently, on the visibility complex of unit balls. This bound improves the trivial bound of $\Omega\left(n^{2}\right)$ for unit balls and also the $\Omega\left(n^{3}\right)$ lower bound for balls of arbitrary radii. This result is particularly surprising because it was natural to conjecture that the visibility complex of fat objects of similar size had a lower worst-case complexity than that for thin triangles. Our result settles negatively this conjecture, and shows exactly the opposite, that is, that fatness and similarity, alone, do not reduce the worst-case complexity of that structure.

Our second result is a worst-case lower bound of $\Omega\left(n^{3}\right)$ on the complexity of the space of free lines among balls of arbitrary radii. This bound improves the trivial $\Omega\left(n^{2}\right)$ bound and almost matches the $O\left(n^{3+\varepsilon}\right)$ upper bound recently proved by Rubin [24]. 
Known worst-case complexities of sets of free lines and maximal free line segments are summarized in Table 1 .

Paper organization. We prove in Section 2 the $\Omega\left(n^{3}\right)$ lower bound on the complexity of the space of free lines among $n$ balls. In Section 3, we prove the bound of $\Theta\left(n^{4}\right)$ on the space of maximal free line segments among $n$ unit balls.

We will describe our lower-bound constructions using a Cartesian coordinate system $(x, y, z)$. In this coordinate system, we often denote by $M_{x}, M_{y}$ and $M_{z}$ the coordinates of a point $M$ (or also the coordinates of the center of a ball $M$ ).

\section{Free lines tangent to balls}

We prove here the following result.

Theorem 1. The combinatorial complexity of the space of free lines among $n$ disjoint balls is $\Omega\left(n^{3}\right)$ in the worst case.

We prove Theorem 1 with a lower-bound construction. For convenience, our construction involves $3 n+3$ balls instead of just $n$, which does not affect the asymptotic complexity.

Refer to Figure 2. We define a set $\mathcal{S}$ of disjoint balls that consists of the following three subsets of $n+1$ balls. We consider first a set of unit balls $\mathcal{B}=\left\{B_{0} \ldots B_{n}\right\}$ whose centers are aligned along the $x$-axis with coordinates $(3(i-n / 2), 0,0)$. We then consider two sets of balls, $\mathcal{A}^{-}=\left\{A_{0}^{-} \ldots A_{n}^{-}\right\}$ and $\mathcal{A}^{+}=\left\{A_{0}^{+} \ldots A_{n}^{+}\right\}$, of sufficiently small radius $\varepsilon$ and whose centers are aligned on two lines parallel to the $y$-axis in the plane $z=1$. As we will see in Lemma 4 , we require $\varepsilon<\frac{1}{540 n^{2}}$. The center of each ball $A_{i}^{-}$has coordinates $(-3 n, 3(i-n / 2) \varepsilon, 1)$, and $A_{i}^{+}$is its reflection with respect to the $y z$-plane.

We prove Theorem 1 by proving the following bound. A line tangent to a set of balls is said to be isolated if it cannot be moved continuously while remaining tangent to these balls.

Proposition 2. There are $\Omega\left(n^{3}\right)$ isolated free lines that are tangent to four of the balls of $\mathcal{S}$.

The idea of the proof is as follows. Consider only two consecutive balls $B_{i}$ and $B_{i+1}$. We study the lines that are tangent to them close to their north poles (i.e., their points with maximum $z$-coordinate). These lines are almost in the horizontal plane $z=1$. Now, in this plane, the balls in $\mathcal{A}^{-}$and $\mathcal{A}^{+}$form two sets of gates which decompose the set of free lines in $\Omega\left(n^{2}\right)$ connected components defined by the gates the line goes through. On the boundary of each such component, there are lines tangent to one ball of $\mathcal{A}^{-}$and one of $\mathcal{A}^{+}$. There are thus $\Omega\left(n^{2}\right)$ free lines tangent to one ball of $\mathcal{A}^{-}$, one of $\mathcal{A}^{+}$, and two consecutive balls of $\mathcal{B}$. Since this can be done for any two consecutive balls of $\mathcal{B}$, there are $\Omega\left(n^{3}\right)$ free lines tangent to four balls. Moreover, since the centers of these balls are not aligned, these tangents are isolated [4].

We now give a formal proof of Proposition 2. The first step of the proof is to prove the following technical lemma which formalizes the fact that the considered tangent lines to two consecutive balls in $\mathcal{B}$ lie almost in the horizontal plane through their north poles.

Let $\tilde{B}_{0}$ and $\tilde{B}_{1}$ be two unit balls centered at $(0,0,0)$ and $(3,0,0)$ and let $L$ be a line tangent to $\tilde{B}_{0}$ and $\tilde{B}_{1}$ respectively at $M_{0}=\left(x_{0}, y_{0}, z_{0}\right)$ and $M_{1}=\left(x_{1}, y_{1}, z_{1}\right)$ in their northern hemispheres (that is, such that $z_{0}$ and $z_{1}$ are positive). Lemma 3 states, roughly speaking, that, as the $y$-coordinates of $M_{0}$ and $M_{1}$ go to 0 , the $z$-coordinates converge quadratically to 1 . 


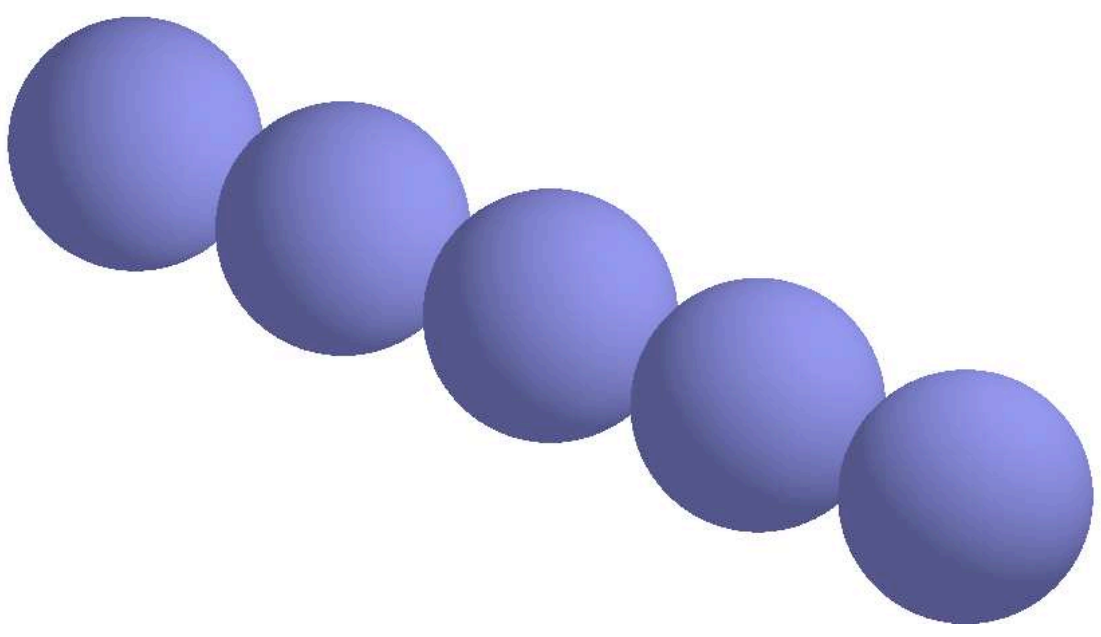

(a) $3 \mathrm{D}$ view.

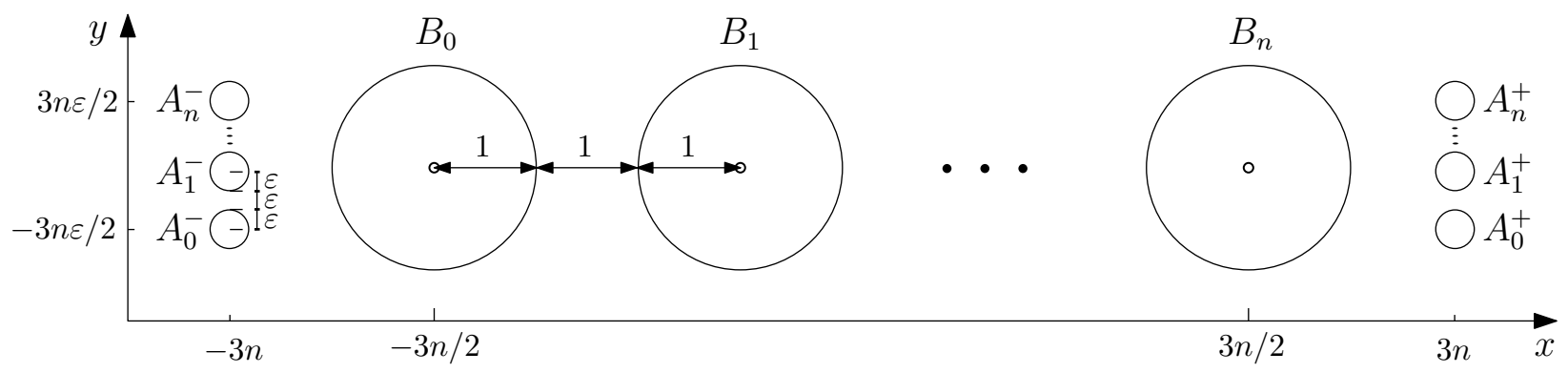

(b) Projection on the $x y$-plane.

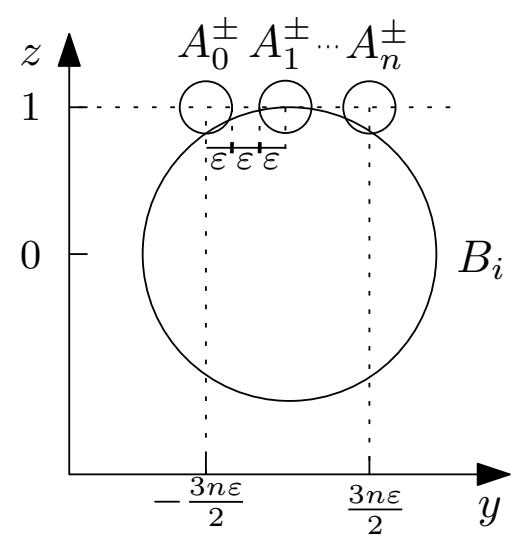

(c) Projection on the $y z$-plane.

Figure 2: Illustration of our construction for Theorem 1 . 


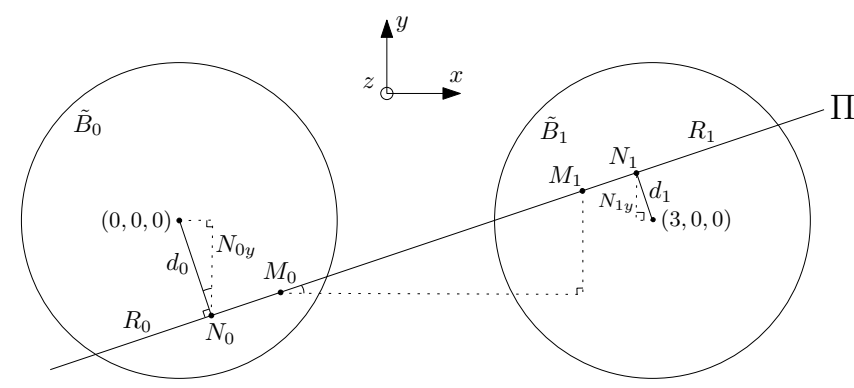

Figure 3: For the proof of Lemma 3: balls $\tilde{B}_{0}$ and $\tilde{B}_{1}$ viewed from above.

Lemma 3. If $\left|y_{0}\right|$ and $\left|y_{1}\right|$ are smaller than some constant $m<1 / 15$, then $z_{0}$ and $z_{1}$ are larger than $1-100 m^{2}$ and $\left|z_{1}-z_{0}\right|$ is smaller than $2 m \cdot\left|y_{1}-y_{0}\right|$.

Proof. We first argue that the result is intuitively clear by showing that it would be straightforward if, instead of balls, we had discs parallel to the $y z$-plane. Writing that $M_{i}$ is on $\tilde{B}_{i}$ gives $x_{0}^{2}+y_{0}^{2}+z_{0}^{2}=$ 1 and $\left(x_{1}-3\right)^{2}+y_{1}^{2}+z_{1}^{2}=1$. Considering discs instead of balls (that is $x_{0}=0$ and $x_{1}=3$ ) gives $\left|z_{i}\right|=\sqrt{1-y_{i}^{2}} \geqslant \sqrt{1-m^{2}} \geqslant 1-m^{2}>1-100 m^{2}$. Furthermore, the difference of the two equations gives $\left|z_{1}-z_{0}\right|=\frac{\left|y_{1}+y_{0}\right| \cdot\left|y_{1}-y_{0}\right|}{\left|z_{1}+z_{0}\right|}<\frac{2 m}{2\left(1-m^{2}\right)} \cdot\left|y_{1}-y_{0}\right|$ which is less that $2 m \cdot\left|y_{1}-y_{0}\right|$ because $\frac{1}{2\left(1-m^{2}\right)}<1$ since $m<1 / 15$.

Since the balls are not discs, we need a few more steps. Consider the vertical plane $\Pi$ that contains $L$ and refer to Figure 3 . Plane $\Pi$ cuts the two spheres in two circles of centers $N_{0}$ and $N_{1}$ and radii $R_{0}$ and $R_{1}$. Let $d_{i}$ denote the signed distance from the center of $\tilde{B}_{i}$ to $\Pi$ (that is to $N_{i}$ ) such that $d_{i}$ has the same sign as $N_{i y}$, the $y$-coordinate of $N_{i}$.

The proof is organized as follows. To prove the first inequality of the lemma (Claim 3), that is that $z_{i}$ is close to 1 , we first show that $\left|d_{i}\right|$ is small (Claim 1). This directly implies that $R_{i}$ is close to 1 and it also gives a simple bound on the difference between $R_{0}$ and $R_{1}$ (Claim 2). We then work in plane $\Pi$ (see Figure 4(a)), where this latter bound implies that $\sin \theta$ is small, or also that $\cos \theta$ is close to 1 , and since $z_{i}=R_{i} \cos \theta$, Claim 2 yields the desired bound on $z_{i}$ (Claim 3). Finally, the second inequality of the lemma (Claim 4) follows from the bound on $z_{i}$ (Claim 3) in a similar way as above when we considered disks instead of balls.

Claim 1: $\left|d_{i}\right| \leqslant 10 \mathrm{~m}$. Notice that, since the two angles shown on Figure 3 are equal, they have the same cosine, that is

$$
N_{i y} / d_{i}=\left(x_{1}-x_{0}\right) / \sqrt{\left(x_{1}-x_{0}\right)^{2}+\left(y_{1}-y_{0}\right)^{2}} .
$$

Since $x_{1}-x_{0} \geqslant 1>0$ and $m<1 / 15$, the right-hand expression can be rewritten as

$$
\frac{1}{\sqrt{1+\left(\frac{y_{1}-y_{0}}{x_{1}-x_{0}}\right)^{2}}} \geqslant \frac{1}{\sqrt{1+4 m^{2}}}>\frac{1}{2} \text {. }
$$

We thus have that $\left|d_{i}\right|<2\left|N_{i y}\right|$ and we now show that $\left|N_{i y}\right| \leqslant 5 m$ which will yield the claim. In projection on the $x y$-plane, since $M_{0}$ and $M_{1}$ are on $L$, the absolute value of the slope of the projection of $L$ is $\frac{\left|y_{1}-y_{0}\right|}{\left|x_{1}-x_{0}\right|} \leqslant 2 m$ since $\left|y_{1}-y_{0}\right| \leqslant 2 m$ and $\left|x_{1}-x_{0}\right| \geqslant 1$. Now, $N_{i}$ is in $\Pi$ so its projection on the $x y$-plane is on the projection of $L$. Since $\left|N_{i x}-x_{i}\right| \leqslant 2\left(M_{i}\right.$ and $N_{i}$ are in the 


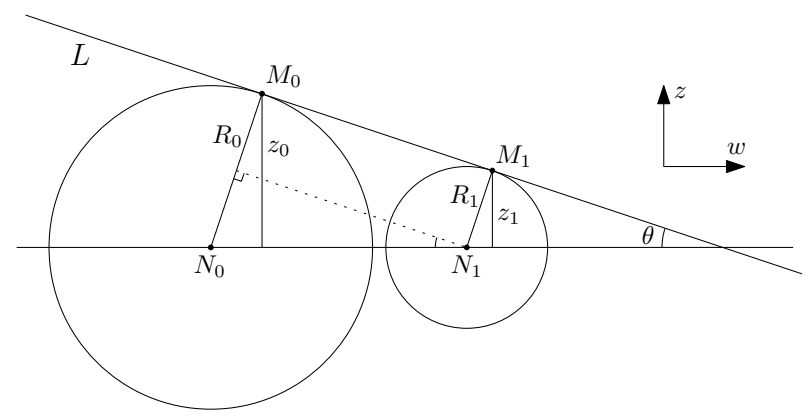

(a)

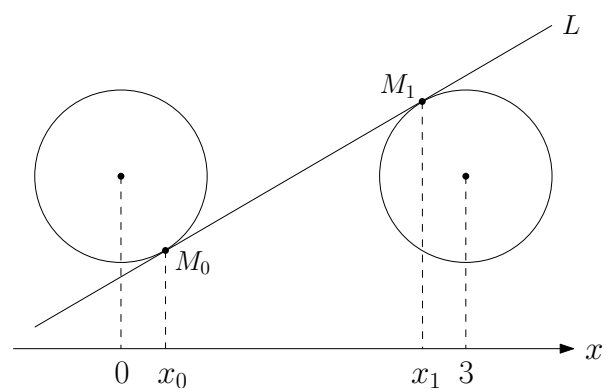

(b)

Figure 4: For the proof of Lemma 3 . (a) intersection of balls $\tilde{B}_{i}$ with plane $\Pi$ (the distance between the disks is not to scale). (b) Intersection of balls $\tilde{B}_{i}$ with plane $\Delta$.

same unit ball), $\left|N_{i y}-y_{i}\right| \leqslant 2 \cdot 2 m$ and thus $\left|N_{i y}\right| \leqslant\left|y_{i}\right|+4 m \leqslant 5 m$, which conclude the proof of Claim 1.

Claim 2: $R_{i} \geqslant \sqrt{1-(10 m)^{2}}$ and $\left|R_{1}-R_{0}\right|<10 m$. The radii of the intersection circles satisfy $d_{i}^{2}+$ $R_{i}^{2}=1$ which implies that $R_{i} \geqslant \sqrt{1-(10 m)^{2}}$ (by Claim 1 ). Since both $R_{i}$ are in $\left[\sqrt{1-(10 m)^{2}}, 1\right]$, $\left|R_{1}-R_{0}\right| \leqslant 1-\sqrt{1-(10 m)^{2}}$ and it is straightforward to prove that $1-\sqrt{1-(10 m)^{2}}<10 m$ is equivalent to $m<1 / 10$ which is satisfied by hypothesis.

Claim 3: $z_{i}>1-100 m^{2}$. We now work in the plane $\Pi$, using a Cartesian coordinate system $(w, z)$ (see Figure 4(a) . Let $\theta$ be the (unsigned) angle between $L$ and the $w$-axis. Consider the line in $\Pi$ parallel to $L$ through $N_{1}$ if $R_{1} \leqslant R_{0}$ and through $N_{0}$ otherwise, as shown on Figure 4(a). Remember that the distance between $N_{0}$ and $N_{1}$ is at least 1 since the balls $\tilde{B}_{0}$ and $\tilde{B}_{1}$ are distance 1 apart. We thus have that $\sin \theta=\left|R_{1}-R_{0}\right| /|| N_{1}-N_{0}||<10 \mathrm{~m}$ by Claim 2, and thus that $\cos \theta>\sqrt{1-(10 m)^{2}}$. Since $z_{i}=R_{i} \cos \theta$ (see Figure $4(\mathrm{a})$ ) and $R_{i} \geqslant \sqrt{1-(10 m)^{2}}$ (Claim 2), we have that $z_{i}>1-(10 m)^{2}$ which is the claim and the second inequality of the lemma.

Claim 4: $\left|z_{1}-z_{0}\right|<2 m \cdot\left|y_{1}-y_{0}\right|$. We first observe that $x_{0}+x_{1}=3$. Indeed, consider the plane $\Delta$ parallel to the $x$-axis and containing line $L$, and refer to Figure 4(b). Since $\Delta$ is parallel to the $x$ axis, it intersects the two balls $\tilde{B}_{0}$ and $\tilde{B}_{1}$ in disks of equal radii whose centers lie, respectively, in the planes $x=0$ and $x=3$. $L$ is also tangent to the two disks in plane $\Delta$ thus, by symmetry, $x_{0}+x_{1}=3$. Now, similarly as at the beginning of the proof, writing that $M_{i}$ is on $\tilde{B}_{i}$ gives $x_{0}^{2}+y_{0}^{2}+z_{0}^{2}=1$ and $\left(x_{1}-3\right)^{2}+y_{1}^{2}+z_{1}^{2}=1$, whose difference is $y_{0}^{2}+z_{0}^{2}-y_{1}^{2}-z_{1}^{2}=0$. Thus, $\left|z_{1}-z_{0}\right|=\frac{\left|y_{1}+y_{0}\right| \cdot\left|y_{1}-y_{0}\right|}{\left|z_{1}+z_{0}\right|}$ and since $\left|y_{i}\right|<m$ and $z_{i}>1-100 m^{2}$ (Claim 3), we have that $\left|z_{1}-z_{0}\right|<\frac{2 m}{2\left(1-100 m^{2}\right)} \cdot\left|y_{1}-y_{0}\right|$ which is less that $2 m \cdot\left|y_{1}-y_{0}\right|$ because $\frac{1}{2\left(1-100 m^{2}\right)}<1$ since $m<1 / 15$. This proves the first inequality of the lemma and concludes the proof.

We now prove that, roughly speaking, a line tangent to two consecutive balls of $\mathcal{B}$ near their north poles intersects each of the convex hulls of $\mathcal{A}^{-}$and of $\mathcal{A}^{+}$and thus that the balls of $\mathcal{A}^{ \pm}$play the role of gates as discussed earlier.

Let $L$ be a line tangent to $B_{i}$ and $B_{i+1}(0 \leqslant i \leqslant n-1)$ at some points with positive $z$ coordinate and let $L^{+}$and $L^{-}$be the points of intersection of $L$ with the planes $x=3 n$ and 


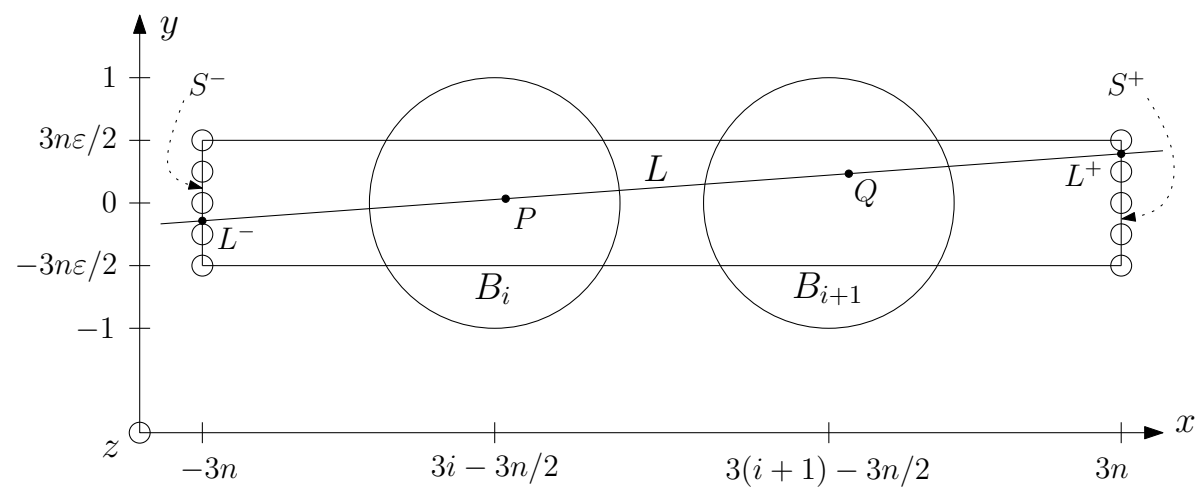

Figure 5: A line $L$ for Lemma 4 .

$x=-3 n$, respectively (see Figure 5).

Lemma 4. If $\left|L_{y}^{+}\right|$and $\left|L_{y}^{-}\right|$are smaller than $3 n \varepsilon / 2$ with $\varepsilon<\frac{1}{540 n^{2}}$, then $\left|L_{z}^{+}-1\right|$ and $\left|L_{z}^{-}-1\right|$ are smaller than $\varepsilon / 2$.

Proof. Let $P$ and $Q$ denote the tangency points of $L$ on $B_{i}$ and $B_{i+1}$ (refer to Figure 5). $L^{-}, P$, $Q$ and $L^{+}$are aligned in this order on $L$, and $\left|L_{y}^{+}\right|$and $\left|L_{y}^{-}\right|$are both smaller than $3 n \varepsilon / 2$, so $\left|P_{y}\right|$ and $\left|Q_{y}\right|$ are smaller than $3 n \varepsilon / 2$. Furthermore, the slope of the projection of $L$ in the $x y$-plane is $\frac{L_{y}^{+}-L_{y}^{-}}{L_{x}^{+}-L_{x}^{-}}=\frac{Q_{y}-P_{y}}{Q_{x}-P_{x}}$ and, by hypothesis, $\left|L_{y}^{+}-L_{y}^{-}\right| \leqslant 3 n \varepsilon, L_{x}^{+}-L_{x}^{-}=6 n$ and $\left|Q_{x}-P_{x}\right| \leqslant 5$, so $\left|Q_{y}-P_{y}\right| \leqslant 5 \varepsilon / 2$. We can now apply Lemma 3 because $\left|P_{y}\right|$ and $\left|Q_{y}\right|$ are both smaller than $m=3 n \varepsilon / 2$ which is smaller than $1 / 15$ since $\varepsilon<1 / 540 n^{2}$. We thus get $\left|Q_{z}-P_{z}\right|<2 \frac{3 n \varepsilon}{2}\left|Q_{y}-P_{y}\right| \leqslant$ $3 n \varepsilon \frac{5 \varepsilon}{2}=\frac{15}{2} n \varepsilon^{2}$ and $Q_{z}>1-100\left(\frac{3 n \varepsilon}{2}\right)^{2}$. Moreover, since $Q_{z} \leqslant 1$, we have $\left|Q_{z}-1\right|<100\left(\frac{3 n \varepsilon}{2}\right)^{2}$.

$L^{-}, P, Q$ and $L^{+}$are still aligned on $L$ and we now consider the slope of the projection of $L$ on the $x z$-plane. Similarly as for the projection on the $x y$-plane, we have: $\frac{L_{z}^{+}-Q_{z}}{L_{x}^{+}-Q_{x}}=\frac{Q_{z}-P_{z}}{Q_{x}-P_{x}}$. By construction, $\left|L_{x}^{+}-Q_{x}\right|<6 n$ and $Q_{x}-P_{x} \geqslant 1$ so

$$
\left|L_{z}^{+}-1\right|-\left|Q_{z}-1\right| \leqslant\left|L_{z}^{+}-Q_{z}\right|<6 n\left|Q_{z}-P_{z}\right|<6 \cdot \frac{15}{2} n^{2} \varepsilon^{2} .
$$

Moreover, since $\left|Q_{z}-1\right|<100 \frac{9}{4} n^{2} \varepsilon^{2}$ and $100 \frac{9}{4}+6 \cdot \frac{15}{2}=270$, we have $\left|L_{z}^{+}-1\right|<270 n^{2} \varepsilon^{2}<\varepsilon / 2$ since $\varepsilon<\frac{1}{540 n^{2}}$. The same holds for $\left|L_{z}^{-}-1\right|$.

We can now prove that there are $\Omega\left(n^{3}\right)$ isolated free lines tangent to four of the balls of $\mathcal{S}$.

Proof of Proposition 2. We prove the proposition by showing that any pair of consecutive balls $B_{i}$, $B_{i+1}(0 \leqslant i<n)$ and any two balls $A_{j}^{-}$and $A_{k}^{+}(j, k \in\{0, \ldots, n\})$ admit at least one common tangent free line.

Notice first that any line tangent to $B_{i}$ and $B_{i+1}$ cannot intersect the interior of any ball $B_{j}$ and thus can only be occluded by a ball in $\mathcal{A}^{ \pm}$.

In the $x y$-plane, consider the two segments $S^{+}$and $S^{-}$defined by $x= \pm 3 n$ and $-3 n \varepsilon / 2<y<$ $3 n \varepsilon / 2$ (see Figure 5); as in Lemma 4, we assume $\varepsilon<\frac{1}{540 n^{2}}$. Any pair of points, one on each of these two segments, defines uniquely a line $L$ that lies in the vertical plane containing these two points and such that $L$ is tangent to $B_{i}$ and $B_{i+1}$ at points in their northern hemispheres (at points 


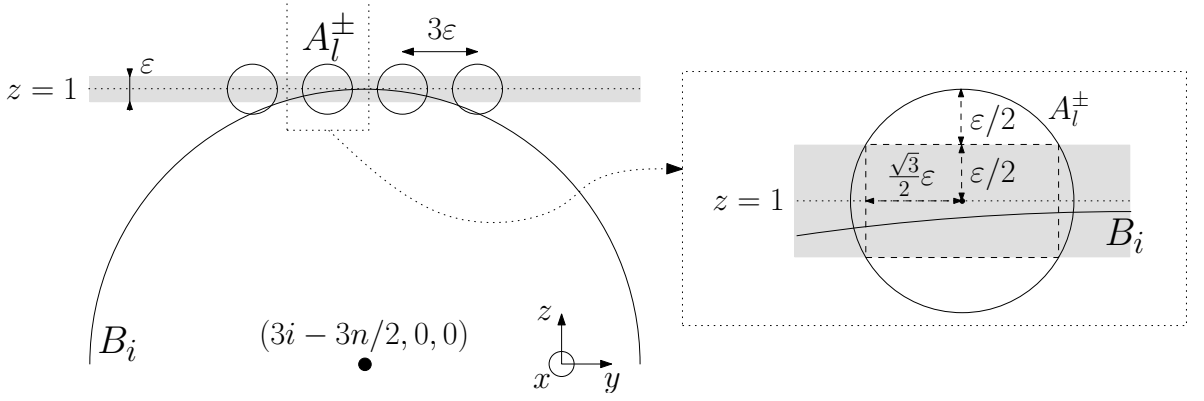

Figure 6: For the proof of Proposition 2; lines $L$ intersect planes $x= \pm 3 n$ in the shaded region.

with positive $z$ coordinates). We parameterize these lines by the $y$-coordinates, $u$ and $v$, of the two points on $S^{-}$and $S^{+}$, respectively, defining the line. In the following, $u$ and $v$ are thus restricted to the interval $[-3 n \varepsilon / 2,3 n \varepsilon / 2]$.

Using this parameterization, we consider the set of lines $L(u, v)$ (or, for simplicity, $L$ ) represented as a square in the $(u, v)$-parameter space. As in the proof of Lemma 4, let $L^{ \pm}$denote the point of intersection of $L$ and plane $x= \pm 3 n$ (note that $u=L_{y}^{-}$and $v=L_{y}^{+}$) and recall that the $y$-coordinate of the center of ball $A_{j}^{-}$is denoted $A_{j y}^{-}$.

We first show that there exist nonempty intervals $I_{j} \subset J_{j}$ of $u$ such that (see Figure 7) the intervals $J_{j}$ are pairwise disjoint and for all $v$ : (i) for all $u \in I_{j}, L(u, v)$ intersects ball $A_{j}^{-}$, (ii) for all $u \notin J_{j}, L(u, v)$ does not intersect ball $A_{j}^{-}$. The same result will also hold by exchanging the roles of $u$ and $v$ and of the $A_{j}^{-}$and $A_{j}^{+}$.

Refer to Figure 6. By Lemma 4, $\left|L_{z}^{-}-1\right|<\varepsilon / 2$. It follows that $\left|L_{y}^{-}-A_{j y}^{-}\right| \leqslant \frac{\sqrt{3}}{2} \varepsilon$ implies that $L$ intersects $A_{j}^{-}$since the squared distance between $L^{-}$and the center of $A_{j}^{-}$is less than or equal to $\left(\frac{1}{2} \varepsilon\right)^{2}+\left(\frac{\sqrt{3}}{2} \varepsilon\right)^{2}=\varepsilon^{2}$. Hence, any line $L(u, v)$ such that $u=L_{y}^{-}$is in $I_{j}=\left[A_{j y}^{-}-\frac{\sqrt{3}}{2} \varepsilon, A_{j y}^{-}+\frac{\sqrt{3}}{2} \varepsilon\right]$ intersects ball $A_{j}^{-}$.

We now show that any line $L(u, v)$ that intersects $A_{j}^{-}$satisfies $u \in J_{j}=\left[A_{j y}^{-}-\frac{5}{4} \varepsilon, A_{j y}^{-}+\frac{5}{4} \varepsilon\right]$. The slope of the projection of line $L$ onto the $x y$-plane is (in absolute value) $\frac{\left|L_{y}^{+}-L_{y}^{-}\right|}{\left|L_{x}^{+}-L_{x}^{-}\right|} \leqslant \frac{3 n \varepsilon}{6 n}=\frac{\varepsilon}{2}$ (see Figure 5 which is less than $\frac{1}{8}$ since $\varepsilon<\frac{1}{540 n^{2}}$. Thus, the $y$-coordinate of points on $L$ varies by at most $\frac{\varepsilon}{4}$ in the slab $-3 n-\varepsilon \leqslant x \leqslant-3 n+\varepsilon$. If $L$ intersects $A_{j}^{-}$, one point of $L$ in this slab has its $y$-coordinate in $\left[A_{j y}^{-}-\varepsilon, A_{j y}^{-}+\varepsilon\right]$, hence $u=L_{y}^{-} \in J_{j}$.

We now partition the set of lines $\mathrm{L}$ in parameter space $(u, v)$ as follows (see Figure 7): the dark gray region is the set of $(u, v)$ such that $u$ or $v$ is in some $I_{j}$; the white region is the set of $(u, v)$ such that neither $u$ nor $v$ belongs to $\bigcup_{j} J_{j}$; the light gray region is the complement of the dark gray and white regions in $\left[\frac{-3 n \varepsilon}{2}, \frac{3 n \varepsilon}{2}\right]^{2}$.

Finally, consider a line $L(u, v)$ for $(u, v)$ in a connected component of the white region bounded by the $u$-strips $J_{j}$ and $J_{j+1}$ and by the $v$-strips $J_{k}$ and $J_{k+1}$ (the hatched region in Figure 7). By the above properties of intervals $I_{j}$ and $J_{j}$, if we decrease $u$ (resp. increase $u$ ), the line $L(u, v)$ while remaining free becomes, at some point in the gray region, tangent to $A_{j}^{-}$(resp. $A_{j+1}^{-}$). Similarly, while $L(u, v)$ remains free and tangent to $A_{j}^{-}$or $A_{j+1}^{-}$, if we decrease (resp. increase) $v$ ( $u$ may vary slightly in order to maintain the tangency), $L(u, v)$ becomes, at some point, tangent to $A_{k}^{+}$ 


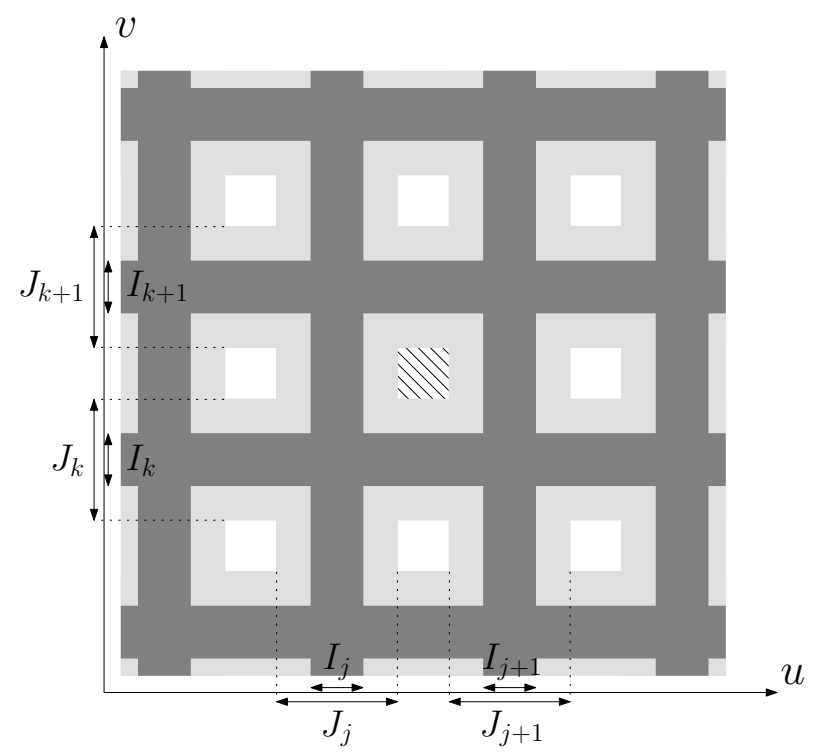

Figure 7: For the proof of Proposition 2: A line parameterized by a point $(u, v)$ in the dark gray region intersects a ball in $\mathcal{A}^{ \pm}$. If $(u, v)$ lies in the white region, the line intersects no ball in $\mathcal{A}^{ \pm}$.

(resp. $\left.A_{k+1}^{+}\right)$. In other words, in parameter space $(u, v)$, the white cell is contained in a connected component of the set of free lines $L(u, v)$ which is bounded by lines $L(u, v)$ that are tangent to $A_{j}^{-}$, $A_{j+1}^{-}, A_{k}^{+}$, or $A_{k+1}^{+}$; moreover, the vertices of the boundary of the cell correspond to lines $L(u, v)$ that are tangent to $A_{j}^{-}$or $A_{j+1}^{-}$and to $A_{k}^{+}$or $A_{k+1}^{+}$.

Hence, any two consecutive balls $B_{i}$ and $B_{i+1}(0 \leqslant i<n)$ and any two balls $A_{j}^{-}$and $A_{k}^{+}$ $(j, k \in\{0, \ldots, n\})$ admit at least one common tangent free line. This concludes the proof because any four balls with nonaligned centers admit finitely many common tangents [4].

Remark. Although our construction admits $\Omega\left(n^{3}\right)$ isolated free lines tangent to four balls, many four-tuples of balls are aligned and thus have infinitely many common tangents. Perturbing all the balls by a sufficiently small amount would easily ensure that all the four-tuples of balls admit finitely many common tangents while all the $\Omega\left(n^{3}\right)$ isolated free lines remain free and tangent to their respective balls.

\section{Free line segments tangent to unit balls}

We prove here the following theorem.

Theorem 5. The combinatorial complexity of the space of maximal free line segments among $n$ disjoint unit balls is $\Theta\left(n^{4}\right)$ in the worst case.

First notice that the $O\left(n^{4}\right)$ upper bound is trivial. We prove the lower bound by giving a construction. Refer to Figure 8 . We define a set $\mathcal{S}$ of disjoint balls that consists of the four subsets $\mathcal{A}^{ \pm}, \mathcal{B}^{ \pm}$of $n$ or $n+1$ balls each. We consider first a set of unit balls $\mathcal{A}^{-}=\left\{A_{1}^{-} \ldots A_{n}^{-}\right\}$whose centers are almost aligned on the $x$-axis, except that each ball is slightly higher than the one in 

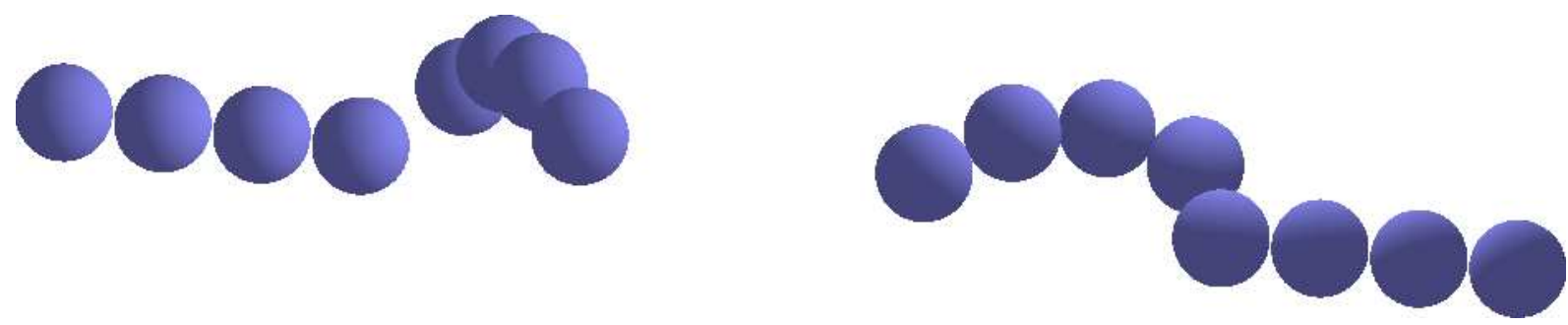

(a) $3 \mathrm{D}$ view.

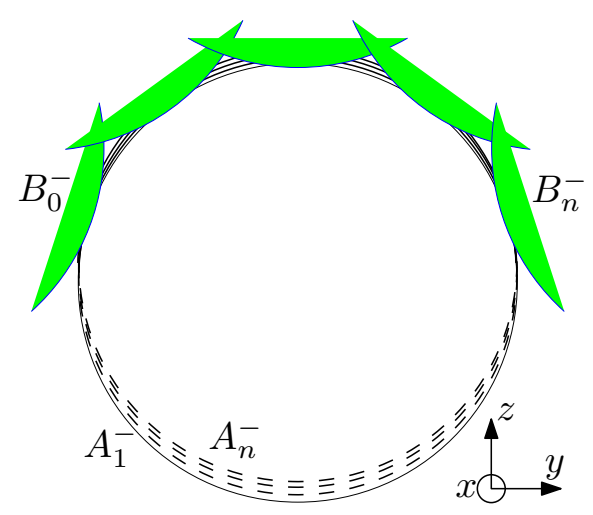

(b) Balls in $\mathcal{A}^{-}$and $\mathcal{B}^{-}$viewed in the $-x$ direction.

Figure 8: Illustration of our construction for Theorem 5 .

front of it (looking from $x=+\infty)$. The center of each ball $A_{i}^{-}$has coordinates $(-M-3 i, 0, i \varepsilon)$ for some large $M$ and some small positive $\varepsilon$. The set $\mathcal{B}^{-}=\left\{B_{0}^{-} \ldots B_{n}^{-}\right\}$consists of unit balls whose centers lie on a helix around the $x$-axis; in particular, the centers project onto the $y z$-plane on a circle centered at the origin and of radius slightly smaller than 2. Note that the purpose of this helix is to ensure that the balls are disjoint; if we allowed intersecting balls, we could simply place all these centers on a circle in the plane $x=-M$. The center of each ball $B_{i}^{-}$has coordinates $\left(-M+3 i,(2-\eta) \sin \left(\alpha_{i}\right),(2-\eta) \cos \left(\alpha_{i}\right)\right)$ where $\alpha_{i}=\alpha\left(-\frac{1}{2}+\frac{i}{n}\right), \alpha$ is a positive constant and $\eta$ is a small positive constant. Finally, the sets $\mathcal{A}^{+}$and $\mathcal{B}^{+}$are the mirror images of $\mathcal{A}^{-}$and $\mathcal{B}^{-}$, respectively, with respect to the $y z$-plane. We consider, in the sequel, $\alpha=\frac{\pi}{4}, \eta=\frac{1}{14 n^{2}}, \varepsilon=\frac{2}{45 n^{3}}$ and $M=1170 n^{3}$.

We prove Theorem 5 by proving the following bound on the balls of $\mathcal{S}$, where a line segment tangent to a set of balls is said to be isolated if it cannot be moved continuously while remaining tangent to these balls.

Proposition 6. There are $\Theta\left(n^{4}\right)$ isolated free line segments that are tangent to four of the balls of $\mathcal{S}$.

The idea of the lower-bound construction is as follows. Consider the affine transformation changing $x$ into $x / M$ which flattens the spheres into ellipsoids. When $M$ tends to infinity, the scene changes (as it depends on $M$ ) and the transformed scene tends to two flat versions of Figure 8(b) on the planes $x= \pm 1$, facing each other. Joining the $\Theta\left(n^{2}\right)$ intersections on each side defines $\Theta\left(n^{4}\right)$ 


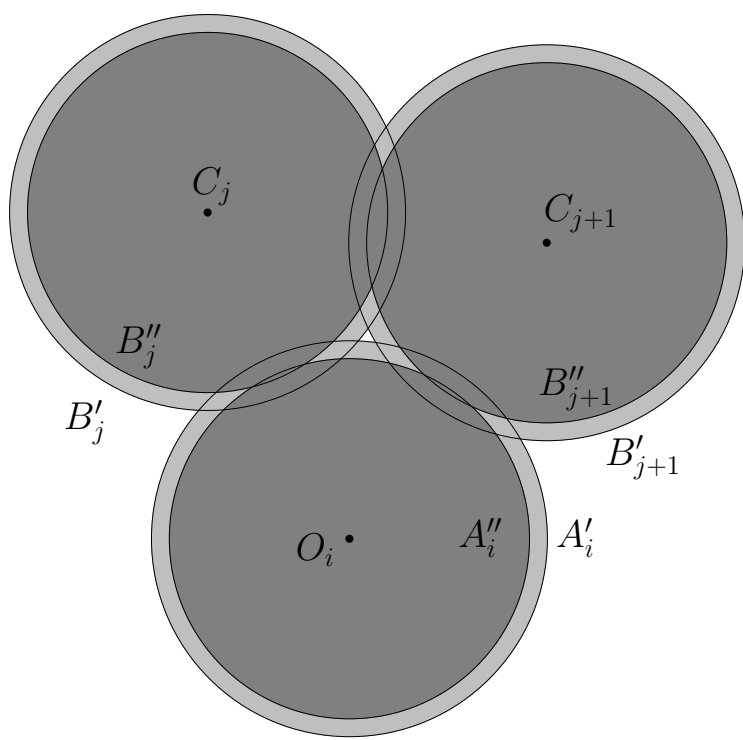

(a)

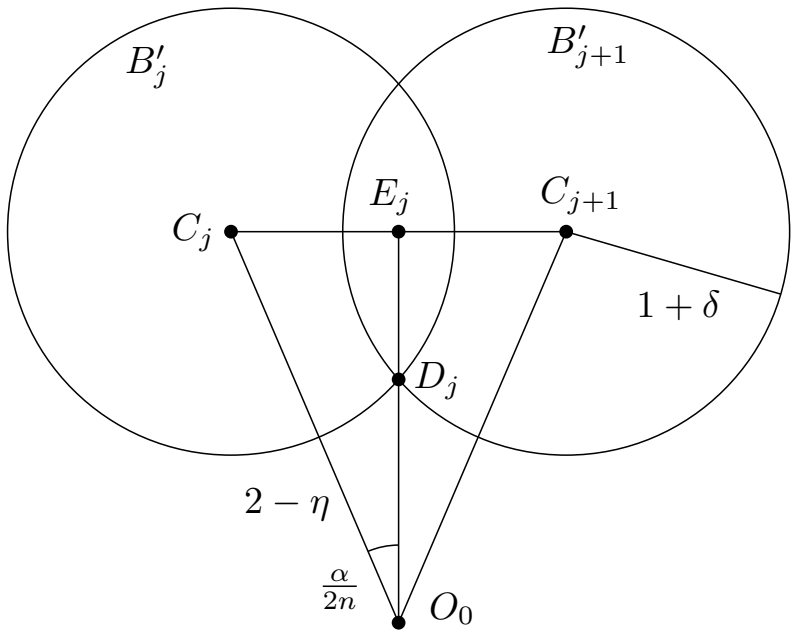

(b)

Figure 9: For the proof of Lemma 7.

free line segments tangent to 4 of the discs. We prove that, for $M$ sufficiently large, the free line segments tangent to 4 of the ellipsoids still exist. Moreover, each of the free line segments tangent to four ellipsoids remains free and tangent to four balls by the inverse affine transformation.

In order to ensure that the set of balls looks like Figure 8(b), $\eta$ and $\varepsilon$ need to be small enough so that, when viewed in the $-x$ direction, the boundary of $A_{i}^{-}$is visible between $B_{j}^{-}$and $B_{j+1}^{-}$. Furthermore, $M$ needs to be large enough so that the view of $\mathcal{A}^{-}$and $\mathcal{B}^{-}$remains combinatorially the same from any point of $\mathcal{A}^{+}$and $\mathcal{B}^{+}$.

We now give a formal proof of Proposition 6, which trivially follows from the next two lemmas.

Lemma 7. Ignoring all the balls of $\mathcal{A}^{-}$but one, $A_{i}^{-}$, and all the balls of $\mathcal{A}^{+}$but one, $A_{i^{\prime}}^{+}$, there are $\Omega\left(n^{2}\right)$ free lines tangent to $A_{i}^{-}, A_{i^{\prime}}^{+}$, a ball of $\mathcal{B}^{-}$and a ball of $\mathcal{B}^{+}$.

Proof. We consider the set of lines $L$ that intersect the plane $x=-M$, in point $L^{-}$, and the plane $x=M$, in point $L^{+}$, such that $-1 \leqslant L_{y}^{ \pm} \leqslant 1$ and $-1 \leqslant L_{z}^{ \pm} \leqslant 2$. Note that this includes all the lines that touch a ball of $\mathcal{A}^{-}$and a ball of $\mathcal{A}^{+}$since $1+n \varepsilon<2$. Between $L^{-}$and $L^{+}$, the $x$-coordinate changes by $2 M$ while the $y$-coordinate changes by at most 2 . Two points of $L$ in the slab $-M-3 n-1 \leqslant x \leqslant-M+3 n+1$ thus have their $y$-coordinates that differ by at most $\frac{2}{2 M}(6 n+2)$. Similarly, the $z$-coordinates differ by at most $\frac{3}{2 M}(6 n+2)$. Hence the projections on $x=-M$ of two points of $L$ in the slab $-M-3 n-1 \leqslant x \leqslant-M+3 n+1$ are at distance at most $\delta=\frac{\sqrt{2^{2}+3^{2}}}{2 M}(6 n+2)<\frac{13 n}{M}=\frac{1}{90 n^{2}}($ for $n \geqslant 2)$.

Let $\tilde{A}_{i}$ and $\tilde{B}_{j}$ be the projections of balls $A_{i}^{-}$and $B_{j}^{-}$on the plane $x=-M$. We construct new disks $A_{i}^{\prime}$ and $B_{j}^{\prime}$ (resp. $A_{i}^{\prime \prime}$ and $B_{j}^{\prime \prime}$ ) of radius $1+\delta$ (resp. $1-\delta$ ) with the same centers as $\tilde{A}_{i}$ and $\tilde{B}_{j}$. We thus have the property that a line $L$ that intersects $A_{i}^{\prime \prime}$ or $B_{j}^{\prime \prime}$ must intersect $A_{i}^{-}$or $B_{j}^{-}$, and a line $L$ that intersects $A_{i}^{-}$or $B_{j}^{-}$must intersect $A_{i}^{\prime}$ or $B_{j}^{\prime}$.

The gist of the proof is that the projections on $x=-M$ look like Figure 9(a), More precisely, 
there is a point on $x=-M$ between $A_{i}^{\prime}, B_{j}^{\prime}$ and $B_{j+1}^{\prime}$ such that any line $L$ through this point is free from $\mathcal{B}^{-}$and $A_{i}^{-}$and $L$ cannot "escape" by a continuous motion without intersecting one of $A_{i}^{-}, B_{j}^{-}$and $B_{j+1}^{-}$. The situation is exactly the same on the other side with $A_{i^{\prime}}^{+}, B_{j^{\prime}}^{+}$and $B_{j^{\prime}+1}^{+}$. We can thus start from a free line $L$, move it down until it is tangent to $A_{i}^{-}$and $A_{i^{\prime}}^{+}$and then keep moving it until is is tangent to $B_{j}^{-}$or $B_{j+1}^{-}$and $B_{j^{\prime}}^{+}$or $B_{j^{\prime}+1}^{+}$.

We now prove that Figure $9(\mathrm{a})$ is a faithful representation. Let $O_{i}$ and $C_{j}$ denote the centers of the discs $\tilde{A}_{i}$ and $\tilde{B}_{j}\left(O_{0}\right.$ has coordinates $(0,0)$ in the $y z$-coordinate system of plane $\left.x=-M\right)$. The distance between $O_{0}$ and $C_{j}$ is $2-\eta$. Using the triangle inequality, the distance between $O_{i}$ and $C_{j}$ is at most $2-\eta+n \varepsilon$. For the discs $B_{j}^{\prime \prime}$ and $A_{i}^{\prime \prime}$ of radius $1-\delta$ to intersect, it is thus sufficient to have $2-\eta+n \varepsilon<2-2 \delta$, i.e., $n \varepsilon+2 \delta<\eta$, which is satisfied here $\left(n \varepsilon=\frac{2}{45 n^{2}}, 2 \delta=\frac{1}{45 n^{2}}\right.$ and $\left.\eta=\frac{1}{14 n^{2}}\right)$.

In order to prove that $A_{i}^{\prime}, B_{j}^{\prime}$ and $B_{j+1}^{\prime}$ have an empty common intersection, we show that the distance between $O_{i}$ and $D_{j}$ is larger than $1+\delta$ where $D_{j}$ is the intersection point of $B_{j}^{\prime}$ and $B_{j+1}^{\prime}$ closest to $O_{0}$ (refer to Figure 9(b) . For convenience, we also consider the midpoint $E_{j}$ of $C_{j}$ and $C_{j+1}$.

$$
O_{0} D_{j}=O_{0} E_{j}-D_{j} E_{j}=(2-\eta) \cos \frac{\alpha}{2 n}-\sqrt{(1+\delta)^{2}-(2-\eta)^{2} \sin ^{2} \frac{\alpha}{2 n}} .
$$

Using the classical inequalities $\sqrt{1+x} \leqslant 1+\frac{x}{2}, \cos x \geqslant 1-\frac{x^{2}}{2}$ and, for $0<x \leqslant \frac{\pi}{16}, \sin x \geqslant x \frac{\sin \pi / 16}{\pi / 16}>$ $\frac{154}{155} x$, and the properties that, for $n \geqslant 2, \eta \leqslant \frac{1}{56}$ and $\delta \leqslant \frac{1}{360}$, we obtain (after factorizing $(1+\delta$ ) outside of the square root):

$$
\begin{aligned}
O_{0} D_{j} & \geqslant(2-\eta)\left(1-\frac{\alpha^{2}}{8 n^{2}}\right)-(1+\delta)+\frac{(2-\eta)^{2}}{1+\delta} \frac{154^{2} \alpha^{2}}{8 \cdot 155^{2} n^{2}} \\
& >1-\eta-\delta-\frac{\alpha^{2}}{4 n^{2}}+\frac{1.982^{2}}{1.003} \frac{154^{2} \alpha^{2}}{8 \cdot 155^{2} n^{2}} \\
& >1-\eta-\delta+0.233 \frac{\alpha^{2}}{n^{2}} .
\end{aligned}
$$

Using the triangle inequality, $O_{i} D_{j} \geqslant O_{0} D_{j}-O_{0} O_{i}$, so

$$
O_{i} D_{j}-(1+\delta)>0.233 \frac{\alpha^{2}}{n^{2}}-\eta-2 \delta-n \varepsilon>\frac{1}{n^{2}}\left(0.14-\frac{1}{14}-\frac{1}{45}-\frac{2}{45}\right)>0,
$$

which concludes the proof that $A_{i}^{-}, A_{i^{\prime}}^{+}, B_{j}^{-}$and $B_{j^{\prime}}^{+}$admit a common tangent, which is free except possibly for the balls of $\mathcal{A}^{ \pm}$.

Lemma 8. For every choice of balls $A_{i}^{-}$and $A_{i^{\prime}}^{+}$, each of the $\Omega\left(n^{2}\right)$ lines of Lemma 7 contains a maximal free line segment tangent to $A_{i}^{-}, A_{i^{\prime}}^{+}$, a ball of $\mathcal{B}^{-}$and a ball of $\mathcal{B}^{+}$.

Proof. For any given balls $A_{i}^{-}$and $A_{i^{\prime}}^{+}$, let $L$ denote one of the $\Omega\left(n^{2}\right)$ lines of Lemma 7 . Notice first that the balls $A_{k}^{-}$, for $k>i$, may intersect $L$ but they cannot intersect $L$ between its tangency points with $A_{i}^{-}, A_{i^{\prime}}^{+}$and the balls $\mathcal{B}^{ \pm}$(recall that all the balls project on the $x$-axis to pairwise distinct intervals).

It thus suffices to prove that, for $k<i, A_{k}^{-}$cannot intersect line $L$. Instead of looking at the slab $-M-3 n-1 \leqslant x \leqslant-M+3 n+1$, we now restrict our analysis to the thinner slab $-M-3 i-1 \leqslant x \leqslant-M-3 k+1$. Similarly as in the proof of Lemma 7, any two points of $L$ in that slab 


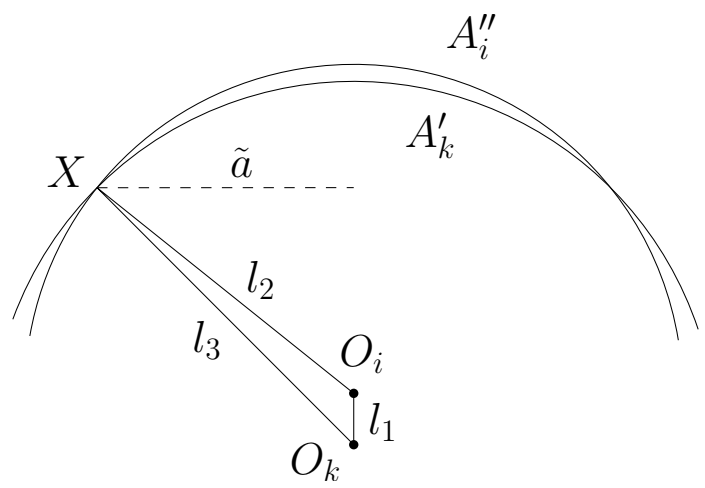

Figure 10: For the proof of Lemma 8

project on the plane $x=-M-3 i-1$ to two points that are at most distance $\delta^{\prime}=\frac{\sqrt{13}}{2 M}(3(i-k)+2)$ apart. The disks $A_{\ell}^{\prime}$ and $A_{\ell}^{\prime \prime}$ are also defined similarly, in the plane $x=-M-3 i-1$, except that $\delta^{\prime}$ is used instead of $\delta$. Let $L_{i}^{-}$denote the intersection point of $L$ with the plane $x=-M-3 i-1$ and recall that $L^{-}$denotes the intersection point with the plane $x=-M$.

We show that in the strip $-a \leqslant y \leqslant a$ of the plane $x=-M-3 i-1$, with $a=2 \sin \frac{\alpha}{2} \approx 0.77$, the upper arc of $A_{i}^{\prime \prime}$ is above $A_{k}^{\prime}$ (see Figure 10. Assuming temporarily that $L$ intersects the plane $x=-M-3 i-1$ in that strip, this proves that $A_{k}^{-}$does not intersect $L$. Indeed, on one hand, since $L$ is tangent to $A_{i}^{-}$, they do not properly intersect, thus $L_{i}^{-}$lies outside disk $A_{i}^{\prime \prime}$, and thus above it; hence $L_{i}^{-}$lies strictly outside disk $A_{k}^{\prime}$. On the other hand, if $L$ intersected $A_{k}^{-}, L_{i}^{-}$would lie inside disk $A_{k}^{\prime}$, a contradiction.

We first show that $L$ does intersect the plane $x=-M-3 i-1$ in the strip $-a \leqslant y \leqslant a$. Temporarily reusing the notations of Lemma 7 and referring to Figure $9(\mathrm{a}),\left|L_{i y}^{-}\right| \leqslant\left|L_{y}^{-}\right|+\delta$, and $L^{-}$ is in the triangle $O_{i} C_{j} C_{j+1}$ so $L_{y}^{-}$is between the $y$-coordinates of $C_{0}$ and $C_{n}$, i.e., $\left|L_{y}^{-}\right| \leqslant(2-\eta) \sin \frac{\alpha}{2}$. As $\delta<\eta \sin \frac{\alpha}{2},\left|L_{i y}^{-}\right| \leqslant a=2 \sin \frac{\alpha}{2}$, which proves the claim.

Now, refer to Figure 10. First notice that for $y=0$, the top of $A_{i}^{\prime \prime}$ is above the top of $A_{k}^{\prime}$ since $2 \delta^{\prime}<(i-k) \varepsilon$. Indeed, $\frac{2 \delta^{\prime}}{(i-k) \varepsilon}=\frac{\sqrt{13}}{M \varepsilon}\left(3+\frac{2}{i-k}\right) \leqslant \frac{5 \sqrt{13}}{M \varepsilon}<\frac{1}{2}$. We thus only need to prove that the two circles do not intersect for $y \leqslant a=2 \sin \frac{\alpha}{2}$. Let $O_{i}$ denote, similarly as before, the projection of the center of $A_{i}^{-}$on $x=-M-3 i-1$, let $X$ be one of the two intersections of $A_{i}^{\prime \prime}$ and $A_{k}^{\prime}$, and let $\tilde{a}=\left|X_{y}\right|, l_{1}=\left\|O_{i} O_{k}\right\|, l_{2}=\left\|O_{i} X\right\|$ and $l_{3}=\left\|O_{k} X\right\|$. We prove that $\tilde{a}>a$. The area of triangle $O_{i} O_{k} X$ can be computed in two ways (the second one uses Heron's formula):

$$
\frac{1}{2} \tilde{a} l_{1}=\frac{1}{4} \sqrt{\left(l_{1}+l_{2}+l_{3}\right)\left(l_{1}+l_{2}-l_{3}\right)\left(l_{1}-l_{2}+l_{3}\right)\left(-l_{1}+l_{2}+l_{3}\right)} .
$$

Recall that $l_{1}=(i-k) \varepsilon, l_{2}=1-\delta^{\prime}$ and $l_{3}=1+\delta^{\prime}$. The factors under the square root are then $(2+(i-k) \varepsilon),\left(-2 \delta^{\prime}+(i-k) \varepsilon\right),\left(2 \delta^{\prime}+(i-k) \varepsilon\right)$ and $(2-(i-k) \varepsilon)$. Since $1 \leqslant i-k \leqslant n$, this yields the inequality $\tilde{a} \geqslant \sqrt{1-\frac{n^{2} \varepsilon^{2}}{4}} \sqrt{1-\left(\frac{2 \delta^{\prime}}{(i-k) \varepsilon}\right)^{2}}$. Since $n \varepsilon<\frac{1}{2}$ and $\frac{2 \delta^{\prime}}{(i-k) \varepsilon}<\frac{1}{2}$, we get $\tilde{a} \geqslant \sqrt{1-\frac{1}{16}} \sqrt{1-\frac{1}{4}} \approx 0.84$. Hence $\tilde{a}>a$, which concludes the proof. 


\section{Conclusion}

We proved a $\Theta\left(n^{4}\right)$ bound on the worst-case combinatorial complexity of the space of maximal free line segments among $n$ balls of unit or arbitrary radii. This closes the problem of bounding the complexity of this space for balls and it improves on the previously known $\Omega\left(n^{3}\right)$ lower bound for balls of arbitrary radii and on the trivial $\Omega\left(n^{2}\right)$ bound for unit balls. This result also settles negatively the natural conjecture that this space of free line segments has smaller worst-case complexity for disjoint fat objects than for skinny triangles.

We also proved an $\Omega\left(n^{3}\right)$ lower bound on the worst-case combinatorial complexity of the space of free lines among $n$ balls of arbitrary radii, improving over the trivial $\Omega\left(n^{2}\right)$ bound. This bound almost matches the upper bound of $O\left(n^{3+\varepsilon}\right)$ from [24] and essentially closes the problem of determining tight worst-case bounds on the complexity of the space of free lines among balls of arbitrary radii. On the other hand, the case of unit balls remains open with a complexity between $\Omega\left(n^{2}\right)$ and $O\left(n^{3+\varepsilon}\right)$ [11, Problem 61].

\section{Acknowledgments}

The authors wish to thank Olivier Devillers and Jeff Erickson for fruitful discussions on this topic. They also thank the anonymous referees for their comments and for noticing that $x_{0}+x_{1}=3$ in the proof of Lemma 3 .

\section{References}

[1] P. K. Agarwal, B. Aronov, V. Koltun, and M. Sharir. Lines avoiding unit balls in three dimensions. Discrete and Computational Geometry, 34(2):231-250, 2005.

[2] P. K. Agarwal, B. Aronov, and M. Sharir. Line transversals of balls and smallest enclosing cylinders in three dimensions. Discrete and Computational Geometry, 21:373-388, 1999.

[3] P. K. Agarwal and M. Sharir. Davenport-Schinzel sequences and their geometric applications. In J.-R. Sack and J. Urrutia, editors, Handbook of Computational Geometry. North-Holland, 1998.

[4] C. Borcea, X. Goaoc, S. Lazard, and S. Petitjean. Common tangents to spheres in $\mathbb{R}^{3}$. Discrete and Computational Geometry, 35(2):287-300, 2006.

[5] H. Brönnimann, O. Devillers, V. Dujmovic, H. Everett, M. Glisse, X. Goaoc, S. Lazard, H. Na, and S. Whitesides. Lines and free line segments tangent to arbitrary three-dimensional convex polyhedra. SIAM Journal on Computing, 37(2):522-551, 2007.

[6] H. Brönnimann, O. Devillers, S. Lazard, and F. Sottile. Lines tangent to four triangles in three-dimensional space. Discrete and Computational Geometry, 37(3):369-380, 2007.

[7] H. Brönnimann, H. Everett, S. Lazard, F. Sottile, and S. Whitesides. Transversals to line segments in three-dimensional space. Discrete and Computational Geometry, 34(3):381-390, 2005 .

[8] B. Chazelle, H. Edelsbrunner, L. Guibas, M. Sharir, and J. Stolfi. Lines in space: combinatorics and algorithms. Algorithmica, 15:428-447, 1996. 
[9] R. Cole and M. Sharir. Visibility problems for polyhedral terrains. Journal of Symbolic Computation, 7(1):11-30, 1989.

[10] M. de Berg, H. Everett, and L. Guibas. The union of moving polygonal pseudodiscs - combinatorial bounds and applications. Computational Geometry: Theory and Applications, 11:69-82, 1998.

[11] E. Demaine, J. Mitchell, and J. O'Rourke. The Open Problems Project. http://maven. smith.edu/ orourke/TOPP/.

[12] J. Demouth, O. Devillers, H. Everett, M. Glisse, S. Lazard, and R. Seidel. On the complexity of umbra and penumbra. Computational Geometry: Theory and Applications, 42(8):758-771, 2009 .

[13] O. Devillers, V. Dujmovic, H. Everett, X. Goaoc, S. Lazard, H.-S. Na, and S. Petitjean. The expected number of 3D visibility events is linear. SIAM Journal on Computing, 32(6):15861620, 2003.

[14] F. Durand. A multidisciplinary survey of visibility, 2000. ACM Siggraph course notes, Visibility, Problems, Techniques, and Applications. http://people.csail.mit.edu/fredo/PUBLI/ surv.pdf.

[15] F. Durand, G. Drettakis, and C. Puech. The 3D visibility complex. ACM Transactions on Graphics, 21(2):176-206, 2002.

[16] A. Efrat, L. J. Guibas, O. A. Hall-Holt, and L. Zhang. On incremental rendering of silhouette maps of a polyhedral scene. Computational Geometry: Theory and Applications, 38(3):129$138,2007$.

[17] D. Halperin and M. Sharir. New bounds for lower envelopes in three dimensions, with applications to visbility in terrains. Discrete and Computational Geometry, 12:313-326, 1994.

[18] H. Kaplan, N. Rubin, and M. Sharir. Line transversals of convex polyhedra in $\mathbb{R}^{3}$. In 20th Annual ACM-SIAM Symposium on Discrete Algorithms - SODA'09, pages 170-179, 2009.

[19] I. Macdonald, J. Pach, and T. Theobald. Common tangents to four unit balls in $\mathbb{R}^{3}$. Discrete and Computational Geometry, 26(1):1-17, 2001.

[20] G. Megyesi and F. Sottile. The envelope of lines meeting a fixed line and tangent to two spheres. Discrete and Computational Geometry, 33(4):617-644, 2005.

[21] G. Megyesi, F. Sottile, and T. Theobald. Common transversals and tangents to two lines and two quadrics in $\mathbb{P}^{3}$. Discrete and Computational Geometry, 30(4):543-571, 2003.

[22] M. Pellegrini. On lines missing polyhedral sets in 3-space. Discrete and Computational Geometry, 12:203-221, 1994.

[23] M. Pocchiola and G. Vegter. The visibility complex. Internat. J. Comput. Geom. Appl., 6(3):279-308, 1996.

[24] N. Rubin. Lines avoiding balls in three dimensions revisited. In 26th Annual ACM Symposium on Computational Geometry - SCG'10, pages 58-67, 2010. 